\title{
In silico Analysis of the Immunological Landscape of Hippocampi in Alzheimer's Disease
}

\author{
Sai Batchu \\ National Heart, Lung, and Blood Institute (NHLBI), Bethesda, MD, USA
}

\section{Keywords}

Dendritic cells · Neuroinflammation - Hippocampi ·

Alzheimer's disease

\begin{abstract}
Introduction: Accumulating evidence suggests a relationship between neuroinflammation and neurodegenerative pathologies such as Alzheimer's disease (AD). This study sought to investigate the immunological composition of hippocampi in patients afflicted with AD. Methods: CIBERSORTX RNA deconvolution was applied on gene expression data developed from hippocampi of 38 AD patients and 17 controls to infer the relative proportions of 22 subsets of immune cells. Results: AD-afflicted hippocampi were found to have greater relative abundances of $\mathrm{M} 2$ macrophages and CD8 T cells. AD-afflicted hippocampi were also composed of significantly more active dendritic cells and significantly fewer resting dendritic cells than control samples. Conclusion: AD-afflicted hippocampi present with a distinct immune signature and dendritic cells may play a critical role in the pathogenesis of $A D$ in this brain component.
\end{abstract}

(c) 2020 S. Karger AG, Basel

\section{Introduction}

Alzheimer's disease (AD), a common cause of neurodegenerative dementia in the elderly, is pathologically characterized by neurofibrillary tangle formation, $\beta$-amyloid deposition creating senile plaques, and neuroinflammation [1]. Although neuroinflammation has been shown to play a critical role, the specifics of immune cell recruitment and infiltration into the brain parenchyma to perpetuate neuroinflammation are only recently being elucidated but are still poorly understood [2-6]. However, the spatiotemporal pattern of neurofibrillary degeneration and progression in $\mathrm{AD}$ has been resolved and starts with brain areas primarily involved in memory and learning, notably the hippocampus [7]. Moreover, there has been no investigation to date specifically examining the total immune cell infiltrate of initially affected brain structures in $\mathrm{AD}$. To address this gap, the study presented herein explored the immune composition of $38 \mathrm{AD}$-afflicted hippocampi through digital cytometry. 


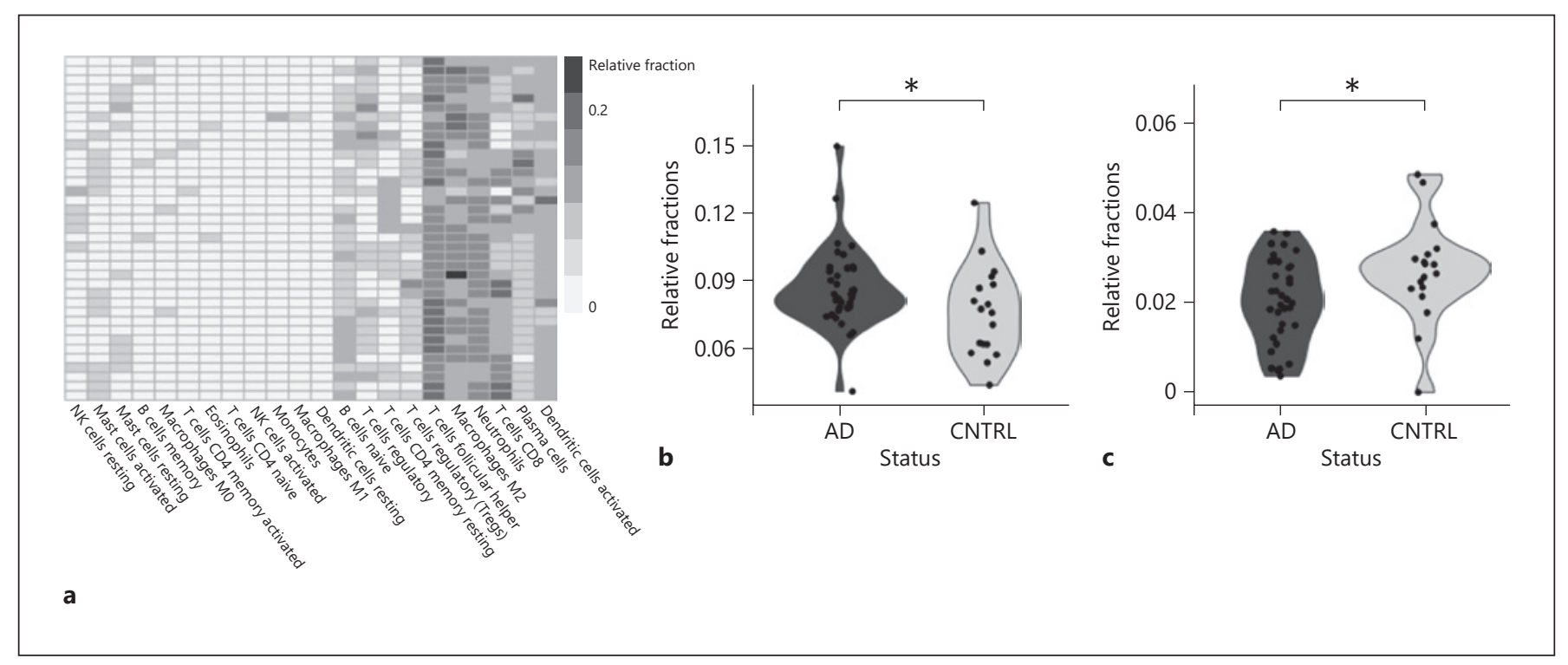

Fig. 1. a Heat map showing relative abundances of immune cell subsets in AD hippocampi. b Violin plot showing relative abundances of active dendritic cells. Asterisk denotes significance at $p<0.05$. c Violin plot showing relative abundances of resting dendritic cells. Asterisk denotes significance at $p<0.05$.

\section{Materials and Methods}

CIBERSORTx, an established in silico RNA deconvolution approach, was used to provide an estimation of the relative abundances of immune cells from a previously published gene expression dataset [8]. The dataset consists of hippocampi gene expression for $38 \mathrm{AD}$ patients and 17 controls [9]. The data was generated using Affymetrix $133 \mathrm{AB}$ platform and was downloaded from Synapse database (https://www.synapse.org/) under accession syn 3157699 .

A validated signature matrix for distinguishing 22 human hematopoietic cell subsets was used to deconvolute the bulk hippocampi gene mixture matrix [8]. The CIBERSORTx algorithm was run with bulk-mode batch correction and quantile normalization with 500 permutations in relative mode. Deconvoluted samples were deemed significant if CIBERSORTx $p$ value was $<0.05$, which represents the significance of the deconvolution results across all cell subsets for goodness of fit [8].

After CIBERSORTx, data was downloaded and analyzed with $\mathrm{R}$ programming language. Normality was not assumed and no values were excluded, thus the nonparametric Mann-Whitney U test was used for comparisons. A $p$ value $<0.05$ was deemed significant.

\section{Results}

After RNA deconvolution for immune cell subset identification, all samples met the $p$ value $<0.05$ cutoffs. Overall, AD hippocampi were enriched mainly for T follicular helper cells, a specialized subset of CD4+ T cells (shown in Fig. 1a). CD8 T cells and M2 macrophages also showed greater relative abundance. When comparing relative abundances between $\mathrm{AD}$ and control samples, $\mathrm{AD}$ hippocampi were significantly enriched for more activated dendritic cells (shown in Fig. 1b) but also had significantly fewer resting dendritic cells (shown in Fig. 1c).

\section{Discussion/Conclusion}

This digital dissection of the immunological landscape from existing gene expression data has shown a distinct immune profile for AD-afflicted hippocampi. Importantly, these results support findings from previous studies. Prior studies have proposed a role for $\mathrm{T}$ follicular helper cells in $\mathrm{AD}[10,11]$ and have shown that increased $\mathrm{T}$ cell and macrophage recruitment into brain parenchyma of $\mathrm{AD}$ patients may modulate $\mathrm{AD}$-associated inflammation $[4,12]$. Furthermore, the novel finding of increased active dendritic cells and decreased resting dendritic cells in hippocampi of $\mathrm{AD}$ patients has not previously been directly characterized. However, prior studies have shown decreased levels of dendritic cells in the blood of AD patients, possibly indicating their recruitment to the brain $[5,13]$.

In conclusion, the unbiased computational approach used herein demonstrated immune infiltrates in $\mathrm{AD}$-afflicted hippocampi, suggesting that dendritic cells, along with other immune cell subtypes, may play a significant 
role in the hippocampi during AD. Further studies examining dendritic cells in $\mathrm{AD}$ are warranted to develop rational therapeutic strategies.

\section{Statement of Ethics}

Ethical approval is not applicable. This article does not contain any studies with human participants performed by the author.

\section{Disclosure Statement}

The author has no conflict of interest to declare.

\section{Funding Sources}

The author has no funding sources to declare.

\section{References}

1 Querfurth HW, LaFerla FM. Alzheimer's disease. N Engl J Med. 2010 Jan;362(4):329-44.

2 Ransohoff RM. How neuroinflammation contributes to neurodegeneration. Science. 2016 Aug;353(6301):777-83.

3 Frost GR, Jonas LA, Li YM. Friend, Foe or Both? Immune Activity in Alzheimer's Disease. Front Aging Neurosci. 2019 Dec;11:337.

4 Gate D, Saligrama N, Leventhal O, Yang AC, Unger MS, Middeldorp J, et al. Clonally expanded CD8 T cells patrol the cerebrospinal fluid in Alzheimer's disease. Nature. 2020 Jan; 577(7790):399-404.

5 Bossù $\mathrm{P}$, Spalletta G, Caltagirone C, Ciaramella A. Myeloid Dendritic Cells are Potential Players in Human Neurodegenerative Diseases. Front Immunol. 2015 Dec;6:632.

6 Serrano-Pozo A, Frosch MP, Masliah E, Hyman BT. Neuropathological alterations in Alzheimer disease. Cold Spring Harb Perspect Med. 2011 Sep;1(1):a006189.
7 Ciccocioppo F, Lanuti P, Pierdomenico L, Simeone P, Bologna G, Ercolino E, et al. The Characterization of Regulatory T-Cell Profiles in Alzheimer's Disease and Multiple Sclerosis. Sci Rep. 2019 Jun;9(1):8788.

8 Newman AM, Steen CB, Liu CL, Gentles AJ, Chaudhuri AA, Scherer F, et al. Determining cell type abundance and expression from bulk tissues with digital cytometry. Nat Biotechnol. 2019 Jul;37(7):773-82.

9 Wang M, Roussos P, McKenzie A, Zhou X, Kajiwara Y, Brennand KJ, et al. Integrative network analysis of nineteen brain regions identifies molecular signatures and networks underlying selective regional vulnerability to Alzheimer's disease. Genome Med. 2016 Nov; 8(1): 104 .

10 Agrawal A, Baulch J, Acharya M, Agrawal S. Identification of peripheral immune mechanisms playing a protective role in Alzheimer's disease progression. J Immunol. 2019 May; 202(Suppl 1):182.36.
11 Baulch JE, Acharya MM, Agrawal S, Apodaca LA, Monteiro C, Agrawal A. Immune and inflammatory determinants underlying $\mathrm{Al}$ zheimer's disease pathology. J Neuroimmune Pharmacol.2020 Feb; https://doi.org/10.1007/ s11481-020-09908-9.

12 Mammana S, Fagone P, Cavalli E, Basile MS, Petralia MC, Nicoletti F, et al. The Role of Macrophages in Neuroinflammatory and Neurodegenerative Pathways of Alzheimer's Disease, Amyotrophic Lateral Sclerosis, and Multiple Sclerosis: Pathogenetic Cellular Effectors and Potential Therapeutic Targets. Int J Mol Sci. 2018 Mar;19(3):831.

13 Ciaramella A, Salani F, Bizzoni F, Orfei MD, Caltagirone C, Spalletta G, et al. Myeloid dendritic cells are decreased in peripheral blood of Alzheimer's disease patients in association with disease progression and severity of depressive symptoms. J Neuroinflammation. 2016 Jan;13(1):18 